\title{
Effects of runway training on behavior in the
}

\section{T-maze}

MITRI E. SHANAB, Fresno State College, Fresno, Calif. 93726, and JOHN W. COTTON, University of California, Santa Barbara, Calif. 93106

Two experimental groups (E1 and E2) were first trained in a numwa before being transferred to a T-maze in which they received the same number of acquisition trials as a control group $(C)$, which had no prior runway training. Group E2 differed from Group E1 in that it received extinction trials in the runway before being transferred to the T-maze. None of the three groups received any pretraining in either apparatus. It was found that Group E2 made significantly more correct choices in the T-maze than either Group EI or Group C. Similarly, Group El was superior to Group C. These results, in conjunction with other similar findings. point to the importance of investigating nunway training as a potent independent variable having marked effects upon T-maze behavior.

The two most extensively used apparatuses in instrumental learning by rats are the runway and the T-maze. There is sufficient evidence in the literature to show that these instruments reveal similar phenomena under comparable conditions. Although certain responses measured in the runway and in the $T$-maze are quite different, little attention has been directed towards finding the effect of receiving training in the one situation upon that in the other situation. For example, what effect does learning in the runway, measured by speed of running, have upon subsequent learning in a $\Upsilon$-maze, measured by per cent of correct choice? If similar tasks are learned in both situations, then acquisition in the runway should facilitate acquisition in the T-maze. This simple prediction is complicated by two further empirical considerations.

First, most animal learning studies include a so-called pretraining period in which animals are first handled (or gentled) and later allowed to explore freely the apparatus in which they are trained later. The purpose of pretraining is to adjust the animal to the $E$ (and, in part, $E$ to the animal), the apparatus, and the special food pellets used as reward. Several studies have shown that handling per se can serve as a potent variable in speeding subsequent learned behavior (Sperling \& Valle, 1964; Denenberg \& Kline, 1964: Du Preez,
1964). Similarly, Shanab (1965) has shown that animals given free (unrewarded) exploration in an elevated Y-maze learned a subsequent discrimination task in the same apparatus significantly faster than a group that received comparable exploration in an elevated straightaway.

Second, Liberman (1951) investigated the degree of positive transfer based upon the acquisition and/or extinction of a response in two kinds of apparatus: the Skinner box and the runway. He found that acquisition of one response did not significantly facilitate acquisition of the other response, although extinction of either response significantly speeded the extinction of the other response.

Liberman used a long period of pretraining (12 days) and a relatively short period of training (eight trials in either acquisition or extinction). The present study proposed to extend Liberman's findings by investigating positive transfer between the runway and the T-maze when no initial pretraining is given and when more training trials are given. Because of the similarity of the responses involved in the runway and the T-maze, it was predicted that there would be positive transfer of running speed from runway to the T-maze. However, on the basis of Liberman's extinction findings, it was expected that $\mathrm{Ss}$ receiving both acquisition and extinction in the runway would make more correct choices in the T-maze than Ss receiving either acquisition or no acquisition in the nunway prior to $T$-maze training. This prediction is based on the assumption that positive transfer would occur on incorrect choices in the T-maze. SUBJECTS

Twenty-four male albino rats, 60 to 80 days old and of the Sprague-Dawley strain, were divided randomly and equally into a control group, $C$, and two experimental groups, E1 and E2. One $S$ in Group C was eliminated for failure to run. APPARATUS

An apparatus was specially built of redwood to serve both as a T-maze and a runway. Two guillotine doors were installed in the stem: one to serve as the exit door for the startbox and the other as the entry door to the runway goalbox. Two gates, made of the same thickness and texture of redwood as the rest of the maze, were used to seal off the arms from the stem. Thus the $T$-maze stem formed the runway. The stem was $4 \mathrm{ft}$ in length from the end of the startbox to the beginning of the runway goalbox. The inside dimensions of the stem and the arms were $9 \mathrm{in}$. in height and 4 in. in width, with the arms being $7 \mathrm{in}$. long. The goalboxes of the $\mathrm{T}$-maze were each $5 \frac{3}{4} \times 13 \times 8 \frac{1}{2}$ in., except for an additional $4 \times 4$ in. recess for placing the food cup where it could not be seen before complete goalbox entry. An automatic timing device was installed to record the S's running time for a distance of $42 \mathrm{in}$. in the stem.

\section{PROCEDURE}

All Ss were housed in individual cages. Each $S$ was handled individually $4 \mathrm{~min}$ a day for 9 days. No pretraining was given. Each $S$ first received 30 acquisition trials (15 trials daily) in its appropriate maze. For Group C, the acquisition trials in the $\mathrm{T}$-maze were followed by 30 extinction trials. Both $E$ groups received acquisition trials in the runway. Following acquisition, Group E1 was given 30 acquisition trials in the T-maze (15 trials daily), while Group E2 received 30 extinction trials in the nunway followed by 30 acquisition trials in the T-maze. Both E1 and E2 received a final series of 30 extinction trials in the $\mathrm{T}$-maze. The $\mathrm{Ss}$ were run in accordance with a prearranged random schedule, so that each $S$ was preceded and followed by an $\mathrm{S}$ from a different group. Reinforcement consisted of a single $45-\mathrm{mg}$ pellet. The minimum intertrial in terval was $15 \mathrm{sec}$, during which time the $S$ had access to water in its living cage. Assignment of the correct goalbox in the T-maze was determined randomly for all $S s$ with the provision that for half the Ss in each group a left turn was correct, while for the other half, a right turn led to the correct goalbox. S was allowed $10 \mathrm{sec}$ on either rewarded or nonrewarded trial.

\section{RESULTS AND DISCUSSION Choice Data}

The course of the acquisition of the discrimination task in the $T$-maze for Groups C, E1, and E2 is depicted in Fig. 1. It is clear that both E1 and E2 had nearly mastered the discrimination by the end of acquisition. The performance of $S s$ in Group C improved slightly over the initial five trials but remained at a level that did not exceed chance expectation $(t=1.78$, $\mathrm{df}=6, p>.05)$. Since most relevant studies show that rats with pretraining master a position task in much less than 30 trials, this atypical performance of Group C must be attributed to the fact that the $S s$ in this group did not receive any pretraining in the apparatus. An analysis of variance performed on the last 15 trials of $T$-maze acquisition yielded a significant group effect ( $F=14.66$, $\mathrm{df}=2 / 20, \mathrm{p}<.001)$. The three possible comparisons among the three groups were 


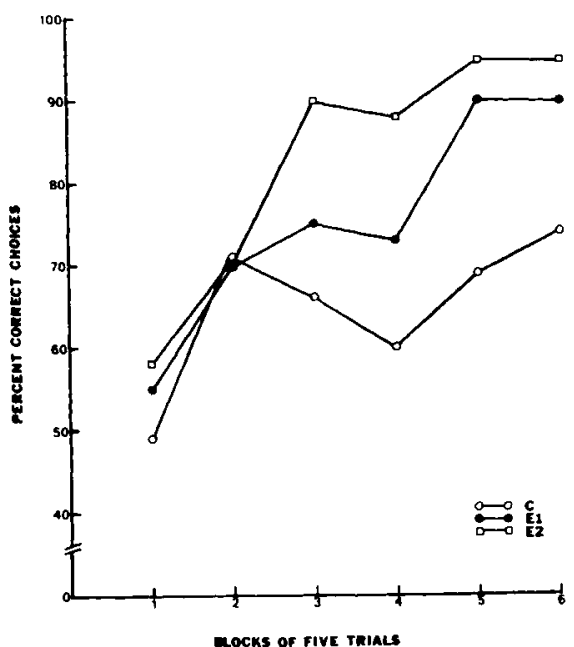

Fig. 1. Percentage of correct choices made in the $T$-maze following zero rewarded, 30 rewarded, and 30 rewarded trials followed by $\mathbf{3 0}$ nonrewarded trials in the runway.

all significant. Thus, Group E2 made significantly more correct choices than did Group E1 $(t=2.273, \mathrm{df}=14, \mathrm{p}<.05)$ as well as Group $C \quad(t=5.041, \quad d f=13$, $\mathrm{p}<.001)$. The difference between Groups $\mathrm{E} 1$ and $\mathrm{C}$ was also significant $(\mathrm{t}=2.818$, $\mathrm{df}=13, \mathrm{p}<.02)$. The superiority of Group E2 over both Groups C and E1 supports the hypothesis that $S$ s receiving rewarded training in the runway followed by extinction in the same runway would learn a subsequent discrimination task in the T-maze faster than Ss given only rewarded training or no training in the runway. The superiority of Group E1 over Group C indicates that Liberman's failure to find positive transfer of acquisition does not hold for the present two apparatuses.

An analysis of variance of the extinction stage choice data (all 30 trials included) revealed a nonsignificant group effect (F $<1)$.

\section{Speed Data}

Acquisition in the T-maze. Figure 2 (left panel) portrays the mean speeds of the three groups during T-maze acquisition, showing much lower speeds for Group C than for the other groups. An analysis of variance performed on the mean speeds over all 30 trials of T-maze acquisition yielded a significant "group effect $(F=38.851$, df $=2 / 20, p<.001)$. Only the differences between Groups $\mathrm{E} 2$ and $\mathrm{C}$

Fig. 2. (Left panel) Mean reciprocal running time in the $T$-maze over six blocks of five trials each following zero rewarded, 30 rewarded, and 30 rewarded followed by 30 nonrewarded trials in the runway. (Right panel) Extinction index in the T-maze for the preceding three conditions (see text). and between Groups $\mathrm{El}$ and $\mathrm{C}$ were significant $(t=6.793, \quad d f=13, p<.001$ and $t=12.138, \mathrm{~d} f=13, \mathrm{p}<.001$, respectively). These findings give further evidence of transfer from the runway task: they differ from the choice data in not showing added improvement due to the extra experiences of Group E2. However, this lack of difference in speed performance between E1 and E2 during $T$-maze acquisition suggests that additional trials per se do not account for the superior choice behavior of E2 over E1. Apparently the effect is due to the fact that E2 had extinction experience in the runway.

Extinction in the T-maze. Since Ss in all groups started extinction with different levels, an extinction index was computed for each $S$ by dividing each extinction score by the mean acquisition speed over the last 15 trials of acquisition. Figure 2 (right panel) also shows the performance of the three groups in extinction using the extinction index as the dependent variable. A two-way analysis of variance was performed in which the three groups formed one variable and the six blocks of five trials each formed the other variable the groups effect was highly significant $(F=17.751, d f=2 / 20, p<.001)$, while the blocks effect was also significant, and the Blocks by Groups interaction was significant $(F=4.893, \mathrm{df}=5 / 100, p<.01$ and $F=2.288, \quad d f=10 / 100, \quad p<.05$, respectively). The apparent superiority of Group C is thus supported by the latter two tests.

Figure 2 further shows that none of the three groups gave any signs of speed decrement during extinction (a result previously obtained in the $T$-maze by Cotton, Lewis, \& Metzger, 1958), since an extinction index of 1.00 means no change between acquisition and extinction (Groups E1 and E2), and an extinction index greater than 1.00 means faster responding in extinction than in acquisition (Group C). This finding is also reminiscent of the overlearning extinction effect (Sperling, 1965), in that both Group E1 and Group E2, which received more training trials than Group $\mathrm{C}$, were less resistant to extinction than Group C.

In summary, it seems that prior acquisition and/or extinction in the runway facilitates subsequent choice behavior in the $T$-maze. Since similar runway training constitutes part of the pretraining procedures used in many experiments, the results point to the importance of investigating more systematically the effects of such pre-experimental procedures upon $\mathrm{T}$-maze behavior. In general, it seems that more attention should be paid to studying the effects of pretraining procedures upon the dependent variables of interest.

\section{REFERENCES}

COTTON, J, W., LEWIS, D. J. \& METZGER, R. Running behavior as a function of apparatus and of restriction of goal box activity. Journal of Comparative \& Physiological Psychology, $1958,51,336-341$.

DENENBERG, V. H., \& KLINE, N. J. Stimulus intensity versus critical periods: A test of two hypotheses concerning infantile stimulation. Canadian Journal of Psychology, 1964, 18, $1-5$.

Du PREEZ, P. D. The persistence of some effects of handling in infancy on the behavior of the adult rat. Quarterly Journal of Experimental Psychology, 1964, 16, 147-155.

LIBERMAN, A. M. A comparison of transfer effects during acquisition and extinction of two instrumental responses. Journal of Experimental Psychology, 1951, 41, 192-198. SHANAB, M. E. Effects of prior exploration on subsequent discrimination learning. Psychonomic Science, 1965, 2, 329-330.

SPERLING, S. E., \& VALLE, F. P Handling-gentling as a positive secondary reinforcer. Journal of Experimental Psychology, 1964, 67, 573-576.

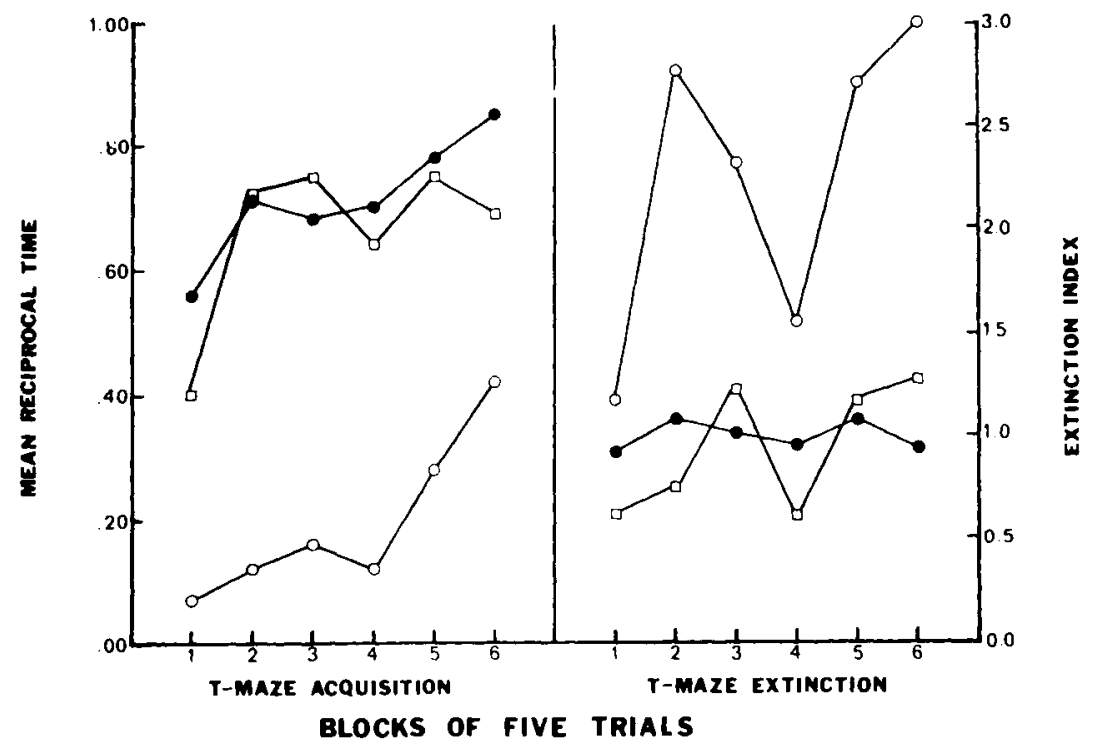

